\title{
The organizational design method for scientific research team based on computational organization theory
}

\author{
Shaohua Wang ${ }^{1,2, a^{*}}$ Lili Rong $^{1}$ \\ ${ }^{1}$ Faculty of Management and Economics, Dalian University of Technology, Dalian 116024, China \\ ${ }^{2}$ Dept. of scientific research, Dalian Naval Academy, Dalian 116018, China \\ ash2680025@126.com
}

Keywords: computational organization theory, organizational design, scientific research team

\begin{abstract}
In order to overcome the disadvantage of current design method for scientific research team, the adaptive organizational design method for scientific research team is put forward based on computational organization theory and modern optimization algorithm. According to the characteristics of scientific research team, the hierarchical description and physical model are used. The organizational design of scientific research team is solved by the use of multidimensional dynamic list scheduling, maximum and minimum cut algorithm and maximal spanning tree algorithm.
\end{abstract}

\section{Introduction}

The optimal organizational design problem is one of finding both the optimal organizational structure (e.g., decision hierarchy, allocation of resources and functions to humans, communication structure, etc.) and strategy (allocation of tasks to decision-makers (DMs), scheduling task execution, detailing decision policies, etc.) that allow the organization to achieve superior performance, while conducting a specific mission[1]. In the field of project management, the organizational design problem of scientific research team has not been paid attention, and in the field of organizational design, this novel organization form of scientific research team has also not been valued, which both lead to the method of organizational design of scientific research team behind the overall development level in the field of project management. Facing the huge project scale and quantity, the scientific research team with traditional organizational structure and design method is inadequate. Organizational contingency theory holds that there are no consistent and optimal organizational structure and form, and the optimal organization is only suitable for the specific conditions. According to this view, the organizational structure design of scientific research team should pay more attention to the influence of its environment and the optimization of organizational design. Only when the organization structure is matched to the environment of scientific research team, the scientific research can demonstrate best performance.

Organizational design includes organizational structure, decision-making process, the resource allocation scheme and the design of incentive mechanism, in which organizational structure design is an important subject in the research of organizational design. The design method of organizational structure can be divided into empirical study, descriptive model and mathematical model. The traditional research is mainly focused on the organizational structure design problems with good conditions and stable environment. In the wake of developments in science and technology, organizational structure design with uncertainty environment has gradually become a hot research. Most of these studies use empirical analysis or descriptive model, and there is less research method based on mathematical model.

In this paper, the disadvantage of current design method for scientific research team is analyzed firstly, and secondly, the organizational design problem of scientific research team is solved by computational organization theory and modern optimization algorithm. Computational organization theory is the study of human organization using computational and mathematical methods[2], whose key is the organization modeling. According to the characteristics of scientific research team, the 
hierarchical description and physical model are used. Thirdly, the adaptive organizational design method for scientific research team is put forward, which is divided into two stages, i.e., goal planning and organization structure design. Finally, the organizational design of scientific research team is solved by the use of multidimensional dynamic list scheduling, maximum and minimum cut algorithm and maximal spanning tree algorithm.

\section{Traditional Organizational Design Method for Scientific Research Team}

The general process of organizational design of scientific research team include that, (1) work division. Specially, decompose organizational tasks according to the rule of consistent goal and efficiency. (2) work classification. It is the foundation of the headship and position in the organization. (3) organizational structure determination. It mainly forms the relationship between different work categories. The specific flow chart is shown in Fig.1.

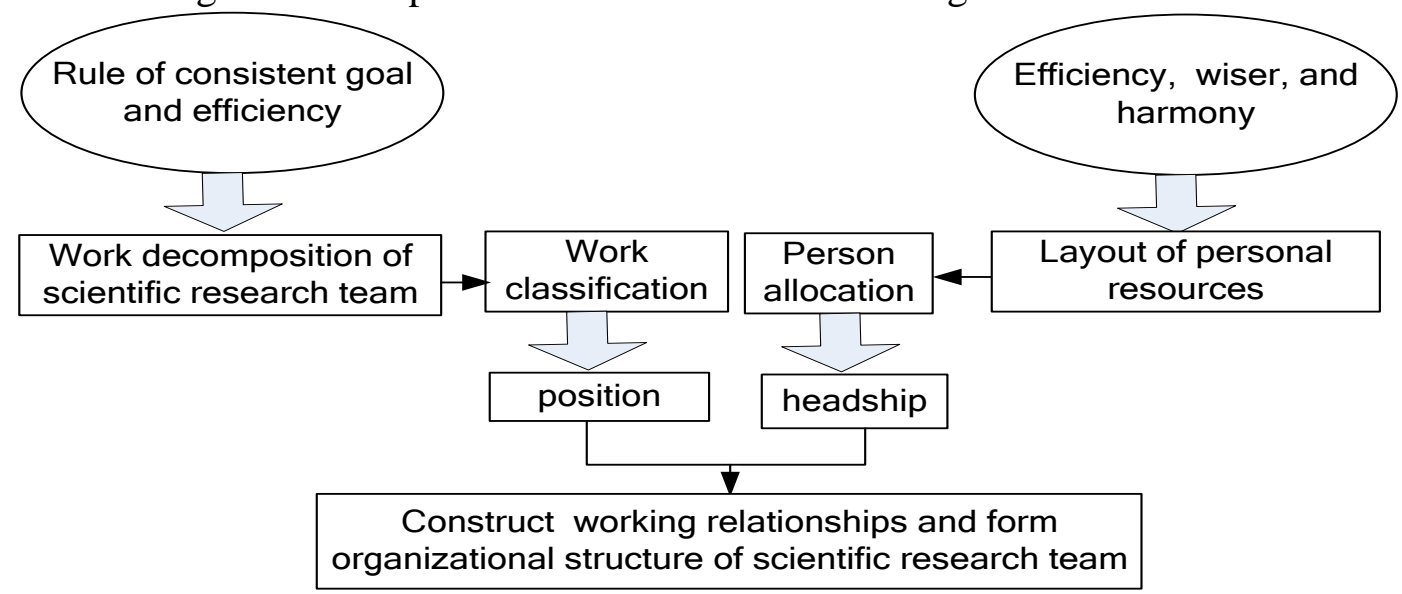

Fig.1 Flow chart of organizational design of scientific research team

The design method of scientific research team can be divided into analysis method and system method[3]. Analysis method means that in order to establish the ideal organizational structure of scientific research team, various factors are coordinated based on organizational design principle, System approach can be regarded as the matching design problem to different organization structure of scientific research team according to the existing structure. Since the organizational structure of scientific research team is usually affected by the project goals, tasks, resources, environment, and so on, the analysis method is relatively difficult to use, and the system approach is obtained more universal application. Both analysis method and system approach are the qualitative analysis method, which depend on the individual talent of organization design personnel and the decision-making ability and personal preference of DMs. Therefore, these two methods cannot exclude the subjective factors and adjust the organizational relationship according to the change of environment. In the development of scientific research team, its environment is complex, and its mission objectives and resource constraints is also changing, especially for large scientific research team. These sources of uncertainty results that it is impossible to predict all kinds of influence factors accurately in the development of scientific research team, then the organizational design according to these factors will show large uncertainties. So the reliability of traditional organizational design is doubtful. In addition, the traditional organizational design cannot realize the coordination of organization structure and its environment. There is only a kind of organization structure, even when the structure is no longer suitable for its environment, it cannot adjust the internal and external work relationship of scientific research team, which will affect the development of scientific research team.

\section{Organizational Description and Model of Scientific Research Team based on Computational Organization Theory}

Organizational Description of Scientific Research Team. Organizational description is the key of computational organization theory. Its main content is to determine the core elements of the 
organization and to describe an organization and its behavior.

Usually, we think that the core elements of organization are the organization members, and an organization can be described through its network of relationships. This traditional thinking makes its computational complexity unbearable when organizational model is established or organizational behavior is analyzed. In fact, apart from the interpersonal relationship network there are the organization constraints between and resources and tasks, hence we can use the network of resources, tasks, personnel relationship to describe an organization. Fig.2 is the schematic diagram of layered organization. An organization is divided into four levels: the organization core, DMs, platform, environment and organizational goals. The organization core is viewed as a virtual organization decision, which is responsible for global information processing, and grasp the organization goal by adjusting the structure among each decision within the organization and the strategy of collaborative task. Decision-making entity is organizational managers or organization operator, which has the power of the platform use and is responsible for specific issues or task execution. Platform is the aggregation of resources. Environment has influence on platform operation, the entity behavior and organizational decision.



Fig. 2 organizational relationships graph

Organizational Model of Scientific Research Team. When environment is changing, the adaptability of scientific research team should be able to make the appropriate adjustments to match its environment. In order to describe the adaptive changes of scientific research team, based on the layered description and adaptive multi-agent system[4], the adaptive organization model is put forward. This model defines and restricts the necessary elements of adaptable organization and describes the adaptive changes of scientific research team under dynamic environment. Specifically, this model contains organization state model, organization structure model and organization transfer equation, i.e.,

$$
\begin{aligned}
& P O=<P O_{\text {state }}, P O_{\text {struct }}, P O_{\text {trans }}> \\
& P O_{\text {state }}=<P, D M, T, F, \text { Rule }, R_{p}, R_{T}, A_{T}> \\
& P O_{\text {struct }}=<R_{p-T}, R_{D M-P}, R_{D M-T}, R_{D M-D M}> \\
& P O_{\text {trans }}: P O^{0} \rightarrow P O^{1}
\end{aligned}
$$

Where $P$ is the platform set, $D M$ is a decision-making entity sets, $T$ is the task set of the organization to achieve, $F$ is the set of the function type owned by organization, Rule is rules set of constraint organization, $R_{p}$ is the number of function types of the platform $P, R_{T}$ is the number of function types required by of tasks $T$,

$$
\begin{gathered}
A_{T}=\left\{\begin{array}{l}
1 \\
0
\end{array} \text { if } \text { The task } T_{i} \text { must be realized before the task } T_{j}\right. \\
R_{P-T}(k, i)= \begin{cases}1 & \text { if } \text { The platform } P_{k} \text { is assigned to } T_{i} \\
0 & \text { else }\end{cases}
\end{gathered}
$$




$$
\begin{gathered}
R_{D M-P}(m, k)= \begin{cases}1 & \text { if } \\
0 & \text { The platform } P_{k} \text { is assigned to } D M_{m}\end{cases} \\
R_{D M-T}(m, i)= \begin{cases}1 & \text { if } \text { The task } T_{i} \text { is assigned to } D M_{m} \\
0 & \text { else }\end{cases} \\
R_{D M-D M}(m, n)= \begin{cases}1 & \text { if } D M_{n} \text { is linked to } D M_{m} \\
0 & \text { else }\end{cases}
\end{gathered}
$$

In the life cycle of scientific research team, facing the dynamic changes of environment, in order to implement adaptive organization transfer in time, it is necessary to establish a complete trigger condition for organization reconstruction. The condition can be divided into three categories: (1) Organizational resource constraints, namely, according to the current organizational structure, whether the allocation of resources is able to finish the current objectives of scientific research team; (2) Organizational performance constraints, i.e., whether the current organization can complete the current goals in certain performance; (3) the changes in the environment of scientific research team.

\section{The Steps of Organizational Design of Scientific Research Team}

The organizational design of scientific research team is based on environment analysis, under the condition of organization constraints (decision-making entity and platform resource constraints), to design the initial state for performing organization goal and the organizational structure which matches this status. The design process includes the following steps:

Step1: environment analysis of scientific research team;

Step2: build the physical model of scientific research team, and determine the constraint parameter of organizational design;

Step3: goal programming. Design the initial state which is adaptive to organizational environment;

Step4: structure design. Design organizational structure which matches to goal programming results.

Environmental analysis of scientific research team is the foundation of determining the parameters of ability and behavior in the physical model, and the environment uncertainty and organizational objectives is the basis of organizational design. Physical model includes entity ability and behavior model and is quantitative description of resources, meanwhile, physical model is the constrained parameters in the structure design. Goal programming is to maximize the goals' effectiveness, and good planning task is the base of structure design. Structure design is the best performance measure for the execution of goal. The process of organization design for scientific research team is shown in Fig.3.

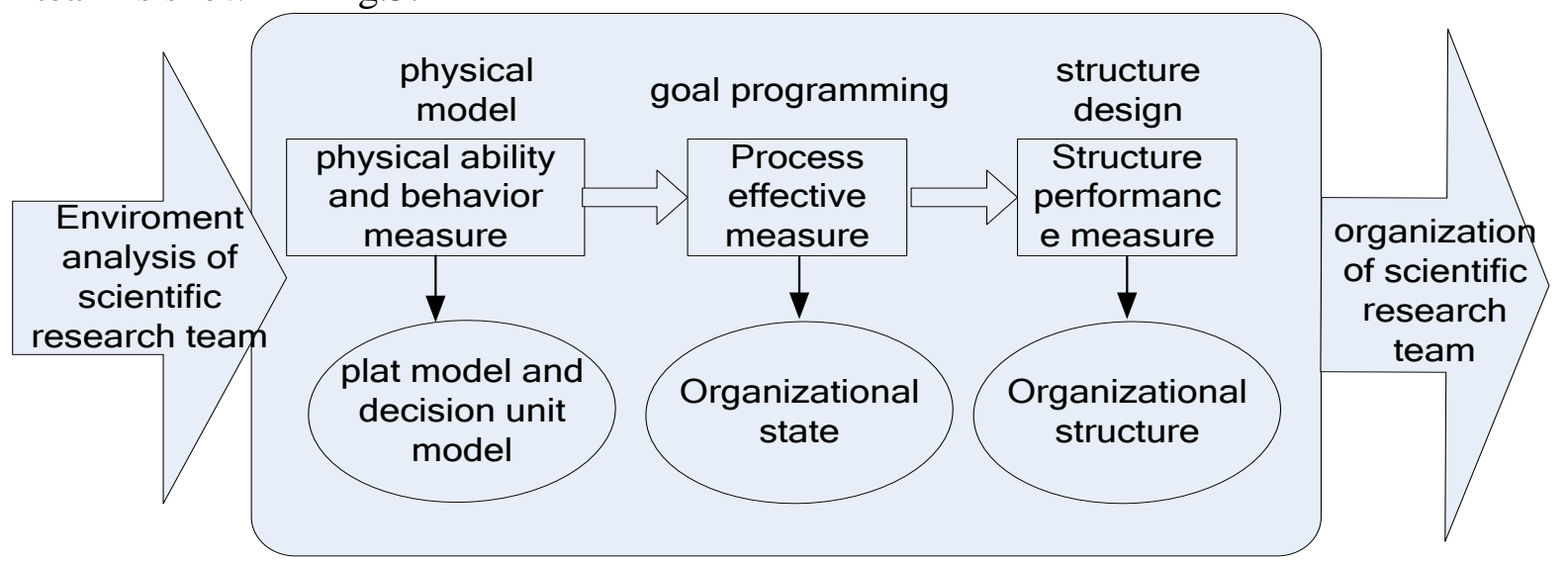

Fig.3 Organizational design process of scientific research team

Various strategies may be utilized to build an organization that accounts for dynamic and 
uncertain mission environments. At one extreme, one may construct an organization capable of processing a range of expected missions. At the other extreme, one may build a finely-tuned organization for a specific mission, and allow online structural reconfiguration and/or strategy adaptation to cope with unforeseen changes in the mission and/or in the organization. The former organizations are able to sustain high levels of performance in dynamic environments without having to alter their structures. The latter organizations are able to generate new strategies and/or reconfigure their structures to potentially achieve even higher performance.

\section{Optimal algorithm for organizational design of scientific research team}

Combining three standard metrics of organization design efficiency, structural design will be divided into mission planning, organizational collaboration net and decision tree design, and each stage needs to adopt different optimization methods to solve.

MDLS Algorithm. The multidimensional dynamic list scheduling (MDLS) finds the platform-task allocation and mission schedule by sequentially assigning tasks to platforms until task set is exhausted. MDLS heuristic has two main steps:

Step1: select the task to be processed.

Step2: select the group of platforms to be assigned to it for processing.

When a task is assigned, platform-task related assignment and scheduling information is updated, as well as the activity coefficients of the platforms. See [5] for details.

Min-max algorithm. Given the data from mission planning, platforms are clustered into groups to be assigned to DMs. The objective is to minimize the DM coordination workload associated with DM-platform-task assignment. The workload is defined as a weighted sum of the internal and direct one-to one external coordination, as well as the task workload. For min-max algorithm, see [5].

Maximal spanning tree algorithm. An alternative is to use maximal spanning tree algorithm to construct the organizational hierarchy tree [6]. The algorithm is as follows:

Step1: select an edge with maximum coordination such that doesn't create cycles in the network. Step2: if ties occur, select the coordination link connected to the DM with minimal workload. Step3: when number of edges in the tree is equal to the number of DM nodes, STOP.

The idea behind the algorithm is that we try to include the largest coordination links and to make DMs with largest workload to be at the lowest level of the hierarchy tree.

\section{Conclusions}

A robust and adaptive organization can maintain an acceptable performance in a changing environment [7]. In order to achieve robustness and adaptability, the organizational design problem of scientific research team is solved by computational organization theory and modern optimization algorithm. The adaptive organizational design method for scientific research team is put forward, which is divided into two stages, i.e., goal planning and organization structure design. Meanwhile, the organizational design of scientific research team is solved by the use of three kinds of optimization algorithm, which indicate multidimensional dynamic list scheduling, maximum and minimum cut algorithm and maximal spanning tree algorithm.

\section{References}

[1] G.M. Levehuk, Y.N. Levehuk, J. Luo, et al, Normative Design of organizations-PartI: Mission Planning, IEEE Transactions on SMC, 32 (2002) 346-359.

[2] K.M. Carley, M.J. Prietula, Computational Organization Theory, Lawrenee Erlbaum Assoeiates Hillsdale NJ, 1994.

[3] Gao Jun, Li Xibin, A study on Design Methodlogy of weaponry Maintenance System, Tianjin People Publishing House, Tianjin ,1998.

[4] SeottA. DeLoaeh, ErieMatson, An Organizational Model for Designing Adaptive Multi-agent Systems, TheAAAI-04 workshop on Agent Organizations: Theory and Practical, (2004) 66-73. 
[5] G.M. Levchuk, Y.N. Levchuk, J. Luo, A library of optimization algorithms for organizational design, the Command and Control Research and Technology Symposium, Monterey, 2000.

[6] G.M. Levehuk, Y.N. Levehuk, J. Luo, et al, Normative Design of Organizations-Part II: Organizational Strueture, IEEE Transactions on SMC, 32(2002) 360-375.

[7] G.M. Levchuk, Y.N. Levehuk, C. Meirina, et al, Normative Design of Organizations-Part III: Modeling Congruent, Robust, and Adaptive Organizations, IEEE Transactions on SMC, 34(2004) 337-350. 